\section{A powerful and personal patient experience}

Align Technology, Inc. (NASDAQ:

ALGN) has announced the launch of its latest digital tool for general dentists and especially Invisalign Go Providers.

The ClinCheck In-face Visualisation tool is an enhanced digital tool, which allows for an image of the patient's face to be incorporated into their treatment plan. The In-face Visualisation tool is now available in the United Kingdom and Ireland.

Actively engaging patients at every stage of their treatment is now simpler than ever before. By helping patients visualise their future smile, general dentists can clearly communicate the benefits of teeth straightening and guide patients towards the treatment choice most suitable for them.

Align Technology's latest enhancement to the 3D Clincheck software will take patient smile visualisation to the next level by helping dentists generate a powerful, clinical visualisation of the patient's teeth in their face. It will also enable general dentists to showcase benefits of teeth straightening and guide their patients towards the most suitable treatment choices. This is made possible with the use of the Invisalign Photo Uploader application (available on iPhone and Android) to capture digital photos of the patient. The iTero intraoral scanner can then capture digital records in a more convenient and efficient way.

Align`s proprietary ClinCheck software has long provided practitioners with $3 \mathrm{D}$ imaging of tooth movements from start to finish so they have options for the patient treatment journey. Now, with the added benefit of the 'In-face' Visualisation, ClinCheck allows dentists to customise treatment plans according to the patient's facial feature to make the visualisation more natural.

The ClinCheck 'In-face' Visualisation tool is now available for general practitioners using the Invisalign Go system in the United Kingdom and Ireland.

For more information, visit www. invisalign-go.co.uk.

\section{Experience the difference}

The EXTARO 300 dental microscope sets itself apart from traditional solutions. It offers a variety of visualisation and capture modes that can be activated with one hand using the system's central Mode Control.

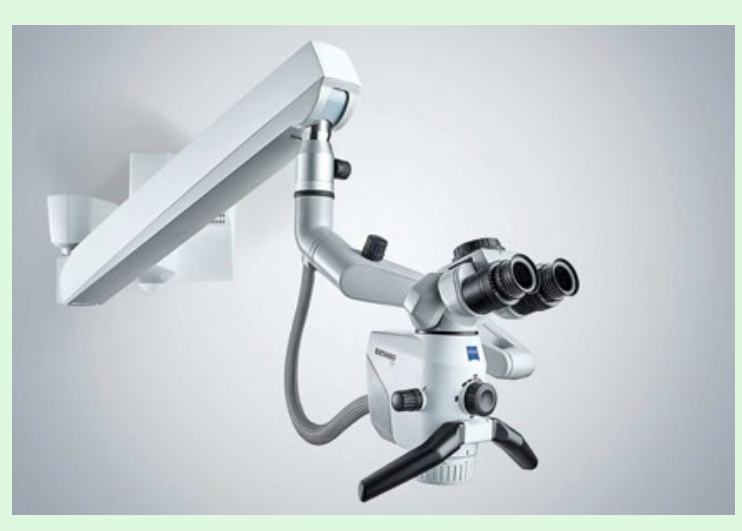

With the same hand, clinicians can adjust the focus of the microscope from their preferred working position, thereby facilitating an uninterrupted workflow. The photo and video capture capabilities of the EXTARO 300 can also be combined with the ZEISS Connect App to highlight problem areas for improved patient education. Experience the difference in treatment with the Carl Zeiss EXTARO 300 dental microscope.

For more information call Nuview on 01453872266 , emailinfo@nuview-ltd.com, visit www.nuview.co or 'like' Nuview on Facebook.

\section{International Zygoma ZAGA Centre}

The Centre for Oral-Maxillofacial and Dental Implant Reconstruction - based at the Manchester ICE Hospital - has been appointed as one of only 20 international ZAGA centres around the world.

The ZAGA method (Zygoma AnatomyGuided Approach) was developed by Dr Carlos Aparicio for reconstruction of the severely atrophic maxillary jaw, using zygomatic implants and avoiding the need for bone grafting. The concept offers a minimally invasive, 3D guided approach to complex cases.
Professor Cemal Ucer - at the Centre for Oral-Maxillofacial and Dental Implant Reconstruction - has been accredited to join a network of 20 centres worldwide under the leadership of Dr Carlos Aparicio in Barcelona. He will be collaborating with this eminent group of ZAGA clinicians to provide education, training and treatment in the field of zygomatic implants.

Please contact Professor Ucer at ice@ ucer.uk or Mel Hay at mel@mdic.co 01612 371842 .

\section{Facilities to be proud of}

Have you seen the new Colosseum Dental practices? Every single one of our premises has been updated, refurbished or relocated to give all our teams the facilities they need to deliver excellent patient care.

We have redesigned layouts to boost ergonomics, we've introduced digital imaging and rotary endodontics. We have also refreshed and brightened each practice to create a welcoming, calming and professional environment in which patients can seek dental care from their trusted teams.

This is just one way in which we are striving to raise standards at Colosseum Dental.

For more information about Colosseum Dental, please visit www.colosseumdental. co.uk.

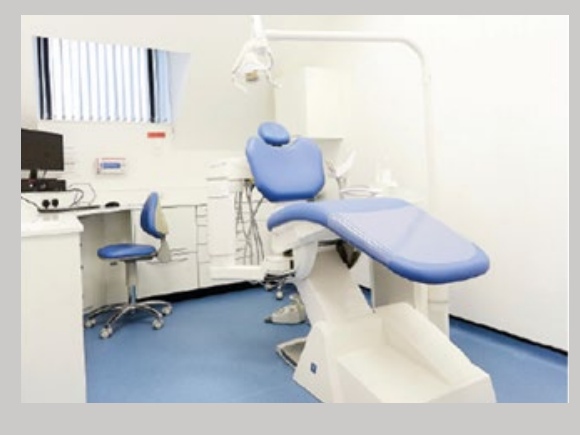

